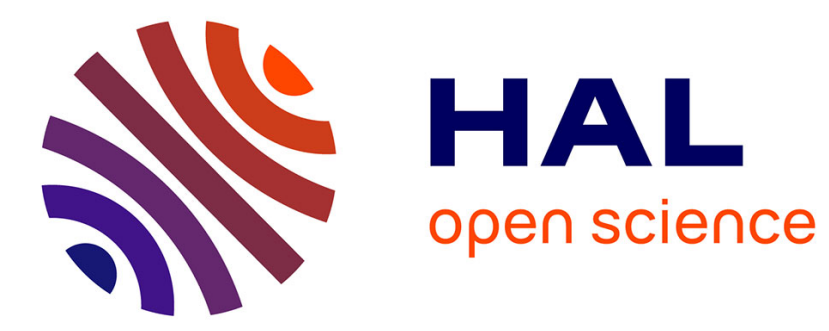

\title{
Prato ou l'exaspération de la diffusion industrielle Évelyne Ritaine
}

\section{To cite this version:}

Évelyne Ritaine. Prato ou l'exaspération de la diffusion industrielle. Sociologie du Travail, 1987, 29

(2), pp.139-156. 10.3406/sotra.1987.2360 . halshs-00105466

\section{HAL Id: halshs-00105466 \\ https://shs.hal.science/halshs-00105466}

Submitted on 26 Apr 2019

HAL is a multi-disciplinary open access archive for the deposit and dissemination of scientific research documents, whether they are published or not. The documents may come from teaching and research institutions in France or abroad, or from public or private research centers.
L'archive ouverte pluridisciplinaire HAL, est destinée au dépôt et à la diffusion de documents scientifiques de niveau recherche, publiés ou non, émanant des établissements d'enseignement et de recherche français ou étrangers, des laboratoires publics ou privés.

\section{(이) $\$$}

Distributed under a Creative Commons Attribution - NonCommercial - NoDerivatives| 4.0 


\title{
Prato ou l'exaspération de la diffusion industrielle
}

\section{Madame Evelyne Ritaine}

\section{Résumé}

L'auteur propose une réflexion sur le dualisme économique des sociétés industrielles en s' interrogeant sur les conditions sociales d'un développement industriel qui serait diffus. L'auteur utilise Prato, ville de Toscane qui, dès le XIVème siècle, est l'aboutissement de réseaux de travail à domicile (- ) pour l'industrie de la laine. L'auteur montre que Prato, en pleine crise du textile, a su tirer parti de son archaïsme industriel en sachant le réactualiser autour des valeurs de métier et de réussite. L'auteur (- ) également avance Vidée d'une logique socio-territoriale dans la diffusion industrielle qui viendrait s'articuler sur le système politico-administratif local.

\begin{abstract}
This reflexion upon the economic dualism of industrial societies inquires into the social conditions of a diffuse industrial development. The example is taken of Prato, a city in Tuscany, Italy, that, since the 14th century, has grown up around networks of home-workers in the wool industry. Despite the textile crisis, Prato has taken advantage of its industrial archaism by bringing it up to date through the values of craftwork and success. There is a social-territorial logic in industrial diffusion that is articulated with the political-administrative system.
\end{abstract}

\section{Citer ce document / Cite this document :}

Ritaine Evelyne. Prato ou l'exaspération de la diffusion industrielle. In: Sociologie du travail, $29^{\mathrm{e}}$ année $\mathrm{n}^{\circ} 2$, Avril-juin 1987 . pp. 139-156:

doi : https://doi.org/10.3406/sotra.1987.2360

https://www.persee.fr/doc/sotra_0038-0296_1987_num_29_2_2360

Fichier pdf généré le 25/05/2018 


\section{Evelyne Ritaine}

\section{Prato ou l'exaspération de la diffusion industrielle*}

L'auteur propose une réflexion sur le dualisme économique des sociétés industrielles en s'interrogeant sur les conditions sociales d'un développement industriel qui serait diffus. L'auteur utilise Prato, ville de Toscane qui, dès le $X I V^{e}$ siècle, est laboutissement de réseaux de travail à domicile (-) pour l'industrie de la laine. L'auteur montre que Prato, en pleine crise du textile, a su tirer parti de son archaïsme industriel en sachant le réactualiser autour des valeurs de métier et de réussite. L'auteur (-) également avance lidée d'une logique socio-territoriale dans la diffusion industrielle qui viendrait s'articuler sur le système politico-administratif local.

* La recherche à Prato a été subventionnée par le ministère des Affaires sociales et de la solidarité nationale (MIRE) dans le cadre de l'appel d'offres " Fractures du social et modes de socialisation ", 1984-1985. Elle a bénéficié d'une subvention complémentaire de la Fondation nationale des sciences politiques. Le travail s'est déroulé au sein du Centre d'étude et de recherche sur la vie locale (Institut d'études politiques de Bordeaux). En Italie l'enquête a reçu l'appui de la Fondazione Feltrinelli (Centro di scienza politica, Prof. Graziano), et du Consorzio centro studi di Prato (dir. M. Romagnoli).

Le débat sur le dualisme économique des sociétés industrielles occupe de plus en plus les économistes et devrait de plus en plus préoccuper les sociologues et les politistes. Les premiers ont montré que les économies modernes tendent à juxtaposer un secteur de production de masse, où la diminution des coûts est obtenue grâce à la stabilité et à la rigidité de l'organisation, et un secteur dit périphérique, où la production est décentralisée par phases dans de petites unités productives, de façon à conserver la plus grande flexibilité productive possible. Ce second secteur se caractérise par l'absence de la grande organisation et par l'utilisation soit de travail intensif dont il réduit le coût (il concerne alors des secteurs économiques traditionnels ainsi 
revivifiés : textile, confection, ameublement, mécanique, etc.), soit de nouvelles technologies aisément décentralisables (machines-outils, micro-électronique, services informatiques, traitement de textes, etc.). Il est adapté aux nouvelles conditions de la demande sur des marchés très versatiles: il est pertinent lorsque la demande a tendance à être discontinue et non standardisée. Il fait preuve d'une grande capacité d'adaptation : aux phases de la demande (production aisément variable), aux transformations de la demande (reprogrammation rapide de la production), aux innovations techniques et esthétiques (savoir-faire professionnel, forte capacité de décision individuelle du chef d'entreprise, investissement matériel léger). Il constitue un moyen de réduction du coût de production en supprimant le coût de reproduction de la grande organisation, en diminuant le poids des charges sociales, en affaiblissant le pouvoir salarial $\left({ }^{1}\right)$.

Le rôle des seconds serait sans doute d'éclairer la controverse née de ce débat : certains ne voient dans le secteur périphérique qu'un réservoir d'innovations, permettant à la fois aux nouveaux pays industrialisés d'amorcer leur croissance et aux pays industrialisés de faire face à la concurrence des premiers; d'autres n'y voient que régression vers une exploitation sauvage du travail. La controverse, recouvrant les intérêts économiques et idéologiques d'acteurs sociaux antagonistes, a peu de chances d'être tranchée. Du moins tant que des études de cas précises n'en éclaireront pas plus les termes $\left({ }^{2}\right)$. En effet, selon les secteurs industriels, l'ancienneté de la décentralisation et les synergies locales, le secteur périphérique peut s'organiser en systèmes industriels faibles (on a alors plutôt des PME concurrentes entre elles, investissant peu et vivant effectivement de la flexibilité du travail), ou en systèmes industriels forts (on a alors plutôt des PME de haut niveau technologique, coopérant entre elles et avec de grandes firmes, produisant effectivement de l'innovation). Avant de qualifier une expérience de développement industriel diffus, il convient donc de préciser, a minima, les moyens de cette fragmentation productive : utilisation de l'économie formelle décentralisée (réseaux de PME et d'artisans, sous-traitance, travail à domicile), utilisation de l'économie

( $\left.{ }^{1}\right)$ S. Berger, M. J. Piore, Dualismo economico e politica nelle società industriali, Bologna, 11 Mulino, 1982; M. J. Piore, C. F. Sabel, «Italian small business development: lessons for U.S. industrial policy ", in J. Zismam, L. Tyson (ed.), American industry in international competition, Ithaca, London, Cornell University Press, 1983; V. Tanzi, The underground economy in the United-States and abroad, Lexington, Toronto, Lexington Books, 1982; C. Lipsig-Mumme, « La rennaissance du travail à domicile dans les économies développées ", Sociologie du travail, 3/1983, p. $313-335$.

${ }^{2}$ ) Il ne paraît pas fondé de traiter sous les mêmes termes, comme on l'entend souvent faire, la diffusion industrielle italienne (ancienne, structurée socialement, contrôlée), l'exploitation du travail irrégulier des minorités ethniques américaines (par exemple M. P. Smith, R. Tardanico, "Les villes américaines et la nouvelle division internationale du travail ", Anthropologie et sociétés, 2/1985, p. 7-23) et l'économie semi-émergée du quartier du Sentier à Paris par exemple. Ni la part de l'économie souterraine, ni les conditions sociales de ces cas ne sont les mêmes. 
informelle dite souterraine (travail non déclaré; double emploi, heures supplémentaires non comptabilisées), combinaison des deux.

Il faut aussi, surtout, s'interroger sur les conditions sociales de ces expériences. Sans doute peut-on faire l'hypothèse que les cas de régression sociale correspondent à des ensembles sociaux (société globale ou sociétés locales, groupes territorialisés, groupes ethniques) destructurés, ayant peu de capacités de régulation de ces nouveaux rapports de force. A contrario, en effet, lorsque la diffusion industrielle se structure en une véritable mode de production, elle se territorialise en correspondance avec une spécificité sociale et culturelle, historiquement constituée et localisée, réactualisée par ce nouveau type de développement : la réussite de la diffusion industrielle correspond à celle de sociétés locales prédisposées. Si la perturbation sociale que représente souvent, en tout cas dans un premier temps, la décentralisation industrielle (affaiblissement syndical, non-garantie des tarifs et des salaires, vague de mises en chômage, conditions de travail non contrôlées) est supportée, c'est que des structures sociales spécifiques (la famille, les réseaux d'interrelations) permettent d'assurer une autre forme de protection. Si la diffusion industrielle est facilement acceptée, c'est que l'esprit d'entreprise est largement répandu dans ces sociétés marquées par le travail indépendant, le caractère familial de l'entreprise, des pratiques de coopération-concurrence largement communautaires. On a pu dire que dans ces systèmes la position des acteurs d'une transaction compte autant que l'objet de la transaction $\left({ }^{1}\right)$. Il semble donc y avoir des conditions sociales de possibilité à la constitution et au fonctionnement des systèmes de diffusion industrielle : conditions de production sociale tout d'abord puisque ces systèmes semblent se constituer grâce à des structures sociales préexistantes, conditions de reproduction ensuite puisque la loi du marché semble régulée par le mode de fonctionnement de ces sociétés locales.

Pour étudier ces conditions le cas italien représente un exemple particulièrement avancé et structuré de développement de la diffusion industrielle, notamment dans l'Italie centrale et nord-orientale $\left({ }^{2}\right)$. L'environnement local y a permis l'expansion en fournissant une culture professionnelle préexistante, la proximité des financements et des relations industrielles, une régulation sociopolitique de type communautaire. Il reste à observer si la phase de maturité industrielle qui s'amorce trouvera aussi des ressources locales ou si la nécessité de complexifier les circuits d'innovation et d'information, de multiplier les marchés, la fera échapper au localisme $\left({ }^{3}\right)$. Pour l'heure, la

(1) F. Chiarello, "Economia informale, famiglia e reticoli sociali ", Rassegna italiana di sociologia, 2/1983, p. 211-254.

$\left({ }^{2}\right)$ A. Bagnasco, Tre Italia, Bologna, 11 Mulino, 1977; G. Fua, C. Zacchia (a cura di), Industrializzazione senza fratture, Bologna, 11 Mulino, 1983.

$\left({ }^{3}\right)$ Pour une interprétation générale cf. E. Ritaine, "Développement économique et changement social : le cas de l'Italie centrale ", article à paraitre, 1987. Pour des données détaillées (dont de nombreux chiffres inédits), cf. E. Ritaine, Ressources sociales du développement économique : un système industriel localisé, en Italie, rapport pour le ministère des Affaires sociales et de la solidarité nationale (MIRE). Multigraphié CERVL, Institut d'études publiques de Bordeaux, 1985. Voir aussi CENSIS, Il delta del localismo, Roma, CENSIS, 1983. 


\section{Evelyne Ritaine}

diffusion industrielle y est suffisamment organisée pour constituer de véritables systèmes d'entreprises $\left({ }^{1}\right)$, généralement monoproductifs et très précisément territorialisés. Ces systèmes peuvent être pulvérisés sur toute une étendue régionale $\left({ }^{2}\right)$, ou se concentrer autour d'un noyau urbain aux fonctions économiques très complètes : on parle alors de districts industriels $\left({ }^{3}\right)$. L'intégration d'une logique économique et d'une logique socioterritoriale dans la diffusion industrielle permet d'étendre à l'Italie centrale et nord-orientale le concept de systèmes industriels localisés, définis comme des systèmes de concurrence-coopération socio-économiques, fondés sur un ensemble de normes et de règles historiquement constitué, articulés sur la stratification sociale et sur le système politico-administratif locaux $\left({ }^{4}\right)$.

Or, les systèmes de diffusion industrielle ont leur ville mythique, Prato en Toscane, la ville dont la richesse est née des chiffons $\left({ }^{5}\right)$. Prato-Dallas diton $\left(^{6}\right)$, tant la course productiviste y est effrénée; étrange course cependant où sont engagés des milliers d'acteurs, car Prato (200000 habitants) compte 11000 entreprises textiles, avec une moyenne de 4,3 actifs par entreprise. Grâce à une histoire locale singulière la ville a en effet anticipé la décentralisation industrielle italienne de quelques vingt années : le "système Prato " est né dans les années cinquante; il est aujourd'hui le district industriel le plus ancien et le plus structuré de l'Italie. L'organisation de la diffusion industrielle y est raffinée à l'extrême; les succès économiques comme les effets pervers y sont exaspérés, préfigurant sans doute ce que les autres zones de diffusion industrielle ont à affronter ou à éviter. Prato est donc d'abord d'une grande originalité ethnographique: spécificité de la matière première d'origine, extrême fragmentation des opérations productives, rôle de l'impannatore, réactions communautaires. C'est aussi comme un laboratoire où observer, dans leurs excès mêmes, la dynamique et les effets de la diffusion indus-

(1) A. Bagnasco, « La costruzione sociale del mercato : strategie di impresa e esperimenti di scala in Italia ", Stato e Mercato, 1985, 13, p. 9-43; A. Bagnasco, C. Trigilia, Societa e politica nell aree di piccola impresa. 11 caso della Valdelsa, Milano, Angeli, 1985; A. Bagnasco, C. Trigilia, Societa e politica nell aree di piccola impresa. il caso di Bassano, Venezia, Arsenale Editrice, 1984.

$\left({ }^{2}\right)$ Ce sera le cas de l'industrialisation dans les Marches par exemple. S. Brusco, "The Emilian model: productive decentralisation and social integration ", Cambridge Journal of Economics, 1982, 6, p. 167-184.

$\left({ }^{3}\right)$ G. Beccatini, "Dal "settore" industriale al "distretto" industriale. Alcune considerazioni sull'unità di indagine dell'economia industriale ", Rivista di economia e politica industriale, 1/1979, p. 7-21.

( $\left.{ }^{4}\right)$ M. F. Raveyre, J. Saglio, "Les systèmes industriels localisés : éléments pour une analyse sociologique des ensembles de PME industriels, Sociologie du travail, 2/1984, p. 157-176.

${ }^{(5)}$ Stracci (chiffons). Prato recycle des chiffons récupérés dans le monde entier et en tire une laine régénérée.

( ${ }^{6}$ G. Bocca, « Le città che decidono il futuro. (5) Mille fili di ricchezza, cosi Prato tesse la moda ", La Repubblica, février 1985. 
trielle $\left({ }^{1}\right)$. Dans le grossissement des traits que produisent son ancienneté, son ampleur et son originalité, apparaît bien comment la diffusion industrielle s'est organisée grâce à un espace social préexistant et comment elle transforme à son tour cet espace social.

\section{** \\ I - L'HÉRITAGE PRÉINDUSTRIEL RÉACTUALISÉ}

Premier de ses paradoxes, Prato s'illustre dans le secteur particulièrement en crise du textile. Ses performances économiques y sont étonnantes : un chiffre d'affaires augmentant de 30\% par an depuis cinq ans; 2000 milliards de lires à l'exportation, autant sur le marché intérieur en 1984; une diversification rapide des matières premières (de la laine régénérée dominante en 1970 à l'association de la laine régénérée, des récupérations de coupe de la confection (ritagli) et des fibres synthétiques et artificielles en 1981) et des produits (des textiles pour la confection et la bonneterie, aux tissus d'ameublement, non tissés, peaux artificielles, etc.); le tout avec $45 \%$ des actifs du textile dans des entreprises de moins de 10 actifs $\left({ }^{2}\right)$. L'éclat de cette réussite tient, second paradoxe, à des archaïsmes devenus ressources grâce à une conjonction d'effets de conjoncture et d'effets de structure. Ainsi dans les années soixantedix, alors que la nouvelle division internationale du travail obligeait les économies occidentales à une adaptation difficile, Prato disposait déjà de tous les atouts pour réussir : son histoire l'avait disposée à saisir cet enjeu.

( $\left.{ }^{1}\right)$ On s'en tient ici à une analyse descriptive, où l'interprétation n'est qu'entamée : il semble nécessaire en effet de faire avancer nos réflexions sur la diffusion industrielle italienne en l'illustrant de cas concrets. L'enquête à Prato a duré quatre mois. Outre l'analyse des travaux existants (très nombreux, surtout en économie) et une campagne d'entretiens avec des observateurs privilégiés, elle s'est appuyée sur une série de suivis ethnographiques de chacun des groupes socioprofessionnels locaux. Par Prato s'entend l'ensemble du système industriel local, soit une agglomération de 7 communes (le circondario di Prato) dont Prato est la commune-centre.

${ }^{(2)}$ Distribution des établissements et des actifs par taille d'entreprise dans le secteur textileconfection, Circondario di Prato, 1975 (Source : commune de Prato) :

\begin{tabular}{ccc}
\hline Actifs (classe d') & Établissements \% & Actifs \% \\
\hline $0-10$ & 92,2 & 44,2 \\
$11-20$ & 4,0 & 12,6 \\
$21-50$ & 2,7 & 17,8 \\
$51-100$ & 0,7 & 10,6 \\
+100 & 0,4 & 14,8 \\
\cline { 2 - 3 } & 100 & 100 \\
\hline
\end{tabular}


Dans cette zone le travail de la laine était de tradition depuis le XIv ${ }^{\mathbf{e}}$ siècle pour les mezzadri (fermiers) qui le pratiquaient comme un indispensable complément de ressources. L'introduction des importantes innovations techniques du XIX ${ }^{e}$ siècle (métier mécanique, utilisation des chiffons comme matière première) n'élimina pas pour autant l'aspect décentralisé de cette production, effectuée à domicile et commercialisée par un acteur typiquement pratésien : l'impannatore $\left({ }^{1}\right)$. Celui-ci est un intermédiaire commercial qui contrôle les marchés et distribue la tâche aux travailleurs à domicile; généralement occupé déjà à d'autres activités (commerçant, rentier), il n'immobilise jamais de capitaux et peut adapter sans risques son activité aux fluctuations saisonnières; c'est un entrepreneur sans entreprise, proche du marchand médiéval $\left({ }^{2}\right.$ ). Cette production se développa peu à peu sur le marché intérieur et sur le marché moyen-oriental, sans changer son organisation. Ce furent seulement les deux guerres mondiales qui, en ouvrant un marché du textile de basse qualité destiné à l'armée, produisirent une relative concentration des capitaux et de la main-d'œuvre : les premières fabbriche (usines) apparurent ainsi, fonctionnant en parallèle avec la production traditionnelle à domicile. Grâce à ce système mixte, Prato devint le centre national de la laine. La relativement faible concentration de la main-d'œuvre réduisait les coûts et maintenait une conception artisanale du travail; l'impannatore, de par la nature même de son rôle, n'effectuait pas la programmation à long terme qui aurait permis la constitution d'un bassin industriel. La capacité d'adaptation ainsi maintenue permit à Prato d'affronter la grave crise de l'après-guerre, due à la perte des marchés coloniaux ${ }^{3}$ ).

En 1950 les fabbriche furent frappées par la récession : sur 20000 ouvriers, 6000 furent licenciés et $11000 \mathrm{mis}$ au chômage partiel. Les entrepreneurs pratésiens, au lieu d'immobiliser des capitaux pour le renouvellement de l'appareil productif, retrouvèrent le geste de l'impannatore : ils cédèrent une partie des machines (notamment des métiers à tisser) aux ouvriers grâce à un système de location ou d'escompte-travail. Toutes les phases de la production qui pouvaient se dérouler hors usine furent ainsi décentralisées en réseaux de travailleurs à domicile, qui peu à peu se transformèrent en artisans. Tous les indices de fragmentation productive attestent de l'ampleur du phénomène : le rapport métier à tisser en usine/métier à tisser à domicile passa de $4 / 1$ en 1949 à $1 / 6$ en 1954; l'effectif des entreprises travaillant pour le compte de tiers fut multiplié par deux; les impannatori devinrent très nombreux; la taille

( $\left.{ }^{1}\right)$ De panna (le drap), le terme est intraduisible en français.

(2) E. Avigdor, L'industria tessile a Prato, Milano, Feltrinelli, 1961. Les marchands médiévaux célèbres, tel le grand Datini, sont devenus les figures emblématiques de la ville.

$\left({ }^{3}\right)$ Ibid. Cf. aussi Comune di Prato, Qualificazione, innovazione e sviluppo dell economia dell area pratese, Convegno di Artimigno, Prato, 1983. 
moyenne de l'entreprise (en actifs) chuta brutalement $\left({ }^{1}\right)$. L'opération rendit toute son élasticité à la production. Pour produire plus en moins de temps il n'était plus nécessaire d'investir, il suffisait de distribuer du travail à plus de petits producteurs (dar'lavoro); en cas de récession il suffisait de ne pas distribuer de travail (non c'è lavoro). Les fluctuations de la demande et les coûts de production étaient désormais répartis sur une multitude de petits producteurs.

Sur ces bases le système Prato s'est consolidé et complexifié au fil des années. S'est constitué peu à peu, outre le volant permanent de travailleurs à domicile, un important groupe de petits producteurs : artisans travaillant pour le compte de tiers (terzisti), artisans indépendants, PME. Chacune de ces unités productives a sa spécialité parmi les innombrables phases de cette production fragmentée : triage, carbonisation des chiffons, cardage, filature, teinturerie, tissage, finitions et contrôles, confection, bonneterie, commerce de gros, etc. $\left({ }^{2}\right)$. Ces petits producteurs sont concurrents entre eux mais font partie de réseaux de producteurs complémentaires qui se transmettent le produit entre eux. Les marchés leur sont attribués soit par les quelques grandes entreprises textiles (lanifici) $\left({ }^{3}\right.$ ) qui sous-traitent une partie de leur production, soit par les impannatori. Comme dans tous les systèmes d'économie diffuse en Italie, l'économie souterraine (sommerso) a joué un rôle dans la constitution du système. Lorsqu'on sait que les groupes d'artisans se sont formés d'ouvriers et d'employés s'installant à leur compte, parvenant même parfois à créer une PME, on peut penser que l'économie souterraine a permis l'accumulation des ressources nécessaires à l'émergence de ces nouveaux producteurs. Maintenant qu'une grande partie de ces activités ont « émergé ",

Évolution du nombre d'entreprises secteur textile-confection par rapport au nombre d'actifs de 1951 à 1971 ( 13 communes autour de Prato) et 1981 ( 7 communes) (source : ISTAT).

\begin{tabular}{rrrr}
\hline Année & Nombre d'actifs & Nombre d'entreprises & Nombre moyen d'actifs \\
\hline 1951 & 21522 & 815 & 26,4 \\
1961 & 41479 & 7614 & 5,5 \\
1971 & 50295 & 10748 & 4,7 \\
\hline 1981 & 50216 & 11503 & 4,2
\end{tabular}

$\left({ }^{2}\right)$ L'entreprise artisanale est juridiquement définie par le nombre d'actifs (10 au maximum) et par la participation directe du propriétaire et de sa famille à la production. Elle bénéficie d'un régime de comptabilité simplifiée et de possibilités de crédit particulières; la représentation syndicale n'y est pas obligatoire et le licenciement plus aisé. Dans le texte " artisanal " est entendu selon ce critère, contemporain de l'enquête; depuis, une nouvelle disposition législative, entérinant les avantages de ce statut, a remonté la barre de définition à 40 actifs (1985). Sur les étapes du cycle productif à Prato cf. E. Avigdor, op. cit; et Cori, Cortesi, Prato, frammentazione $e$ integrazione di un bacino tessile, Torino, Quaderni delle Fondazione Agnelli, 1977.

$\left({ }^{3}\right)$ Rappelons que sur 11000 entreprises du textile-confection, 8883 ont moins de 10 actifs, et seulement 110 plus de 50 actifs (1975). 
elle constitue surtout un élément de souplesse du système $\left({ }^{1}\right)$ : pratiquée ici sous la forme dominante du travail à domicile non déclaré, mais aussi d'heures de travail supplémentaires non comptabilisées, et sans doute d'une forte évasion fiscale, elle est comme ailleurs difficile à estimer $\left({ }^{2}\right)$; ici, comme ailleurs en Italie, si elle facilite la compétitivité elle ne suffit jamais à l'expliquer au fond.

L'originalité du système tient cependant moins à cette nébuleuse de petits producteurs qu'à la façon dont les fonctions de pilotage du système sont assurées : ce sont les lanifici et les impannatori qui contrôlent le marché des matières premières, les relations avec les banques et le crédit, la prospection et la commercialisation (sauf dans le cas, minoritaire, des artisans indépendants). Entre ces acteurs centraux règne une concurrence imparfaite car ils sont liés par des connexions économiques (associations, accords de concurrence), familiales, politiques. Cette centralité ne fait que s'accentuer dans la phase de maturité industrielle ouverte depuis le début des années soixante-dix : alors que dans les autres régions la diffusion industrielle se met en place à cette époque, Prato en est déjà à restructurer sa décentralisation. Plus le cycle productif se pulvérise, plus il se recompose sur le plan financier par formation de groupes d'entreprises (indépendantes les unes des autres mais contrôlées par la même source financière) et par l'intégration de ceux-ci dans des groupes financiers. Ces derniers contrôlent le secteur vital du crédit et le secteur immobilier, très spéculatif en raison de l'explosion démographique qu'a provoquée l'expansion; au cœur du système ces groupes s'enchevêtrent avec un secteur bancaire omniprésent, notamment avec la toute-puissante Cassa di risparmio. Ce sont ces entrepreneurs et ces groupes qui, au sein de la puissante union patronale (UIP), en sont aujourd'hui à parfaire la communication au sein de la dispersion, en mettant sur pied le premier système télématique au service d'un système productif diffus (1 200 entreprises reliées par vidéotext) $\left({ }^{3}\right)$.

D'une sorte d'archaïsme préindustriel à une forme de postmodernité, pour reprendre une qualification chère aux Italiens, telle semble être la raison de la fascination qu'exerce Prato. Du moins tant qu'on s'en tient à l'organisation économique; en termes sociaux l'appréciation est bien plus délicate. Comment en effet concevoir qu'une société ait pu, sans heurts notables, supporter un changement morphologique aussi considérable? A ttirant à elle une importante main-d'œuvre la ville a connu une véritable explosion démographique : la population de l'ensemble de l'agglomération a augmenté de $83 \%$ de 1951 à

(1) CENSIS, Dal sommerso al post-industriale, Milano, Angeli, 1984.

( ${ }^{2}$ ) Les chiffres les plus raisonnés évaluent l'économie souterraine italienne à $17-20 \%$ de la force de travail et $14-20 \%$ du PNB. Cf. B. Contini, "The second economy in Italia ", in V. Tanzi, op. cit.

(3) A. Macelli, M. Romagnoli, « Aspetti del decentramento produttivo nell' area tessile pratese ", Inchiesta, 1979, 38-39. 


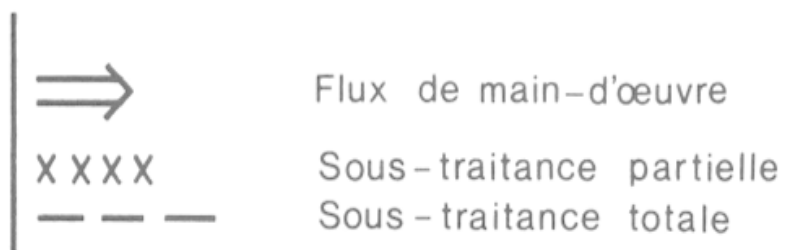

$\square-\square$ Réseaux de producteurs complementaires
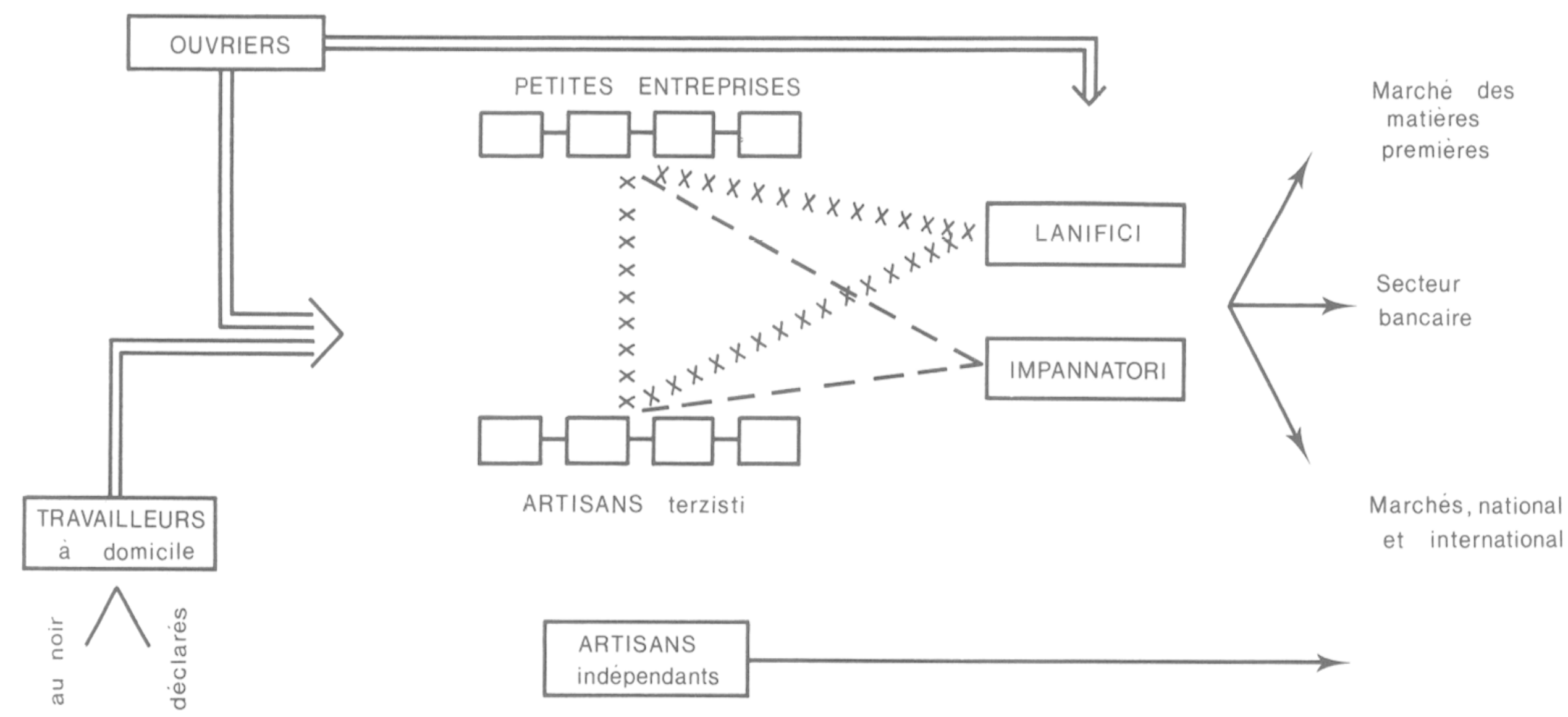

\section{ARTISANS \\ independants}

Les acteurs économiques à Prato 
1980 ; certaines communes ont connu des taux de développement vertigineux (Montemurlo par exemple : $+370 \%$ ) $\left({ }^{1}\right)$. Cette explosion démographique s'est faite par immigration massive : actuellement environ les deux tiers des habitants sont originaires d'une famille ayant immigré à Prato, soit en provenance de la Toscane ( $50 \%$ d'entre eux), soit en provenance du Mezzogiorno et des îles ( $35 \%$ d'entre eux). Dans cette fourmilière humaine, on travaille beaucoup plus qu'ailleurs (les heures de travail déclarées sont supérieures aux moyennes nationales, surtout pour les travailleurs indépendants, et ne tiennent pas compte du travail dissimulé), on s'enrichit plus vite qu'ailleurs aussi $\left({ }^{2}\right)$. Et au milieu de ces bouleversements on demeure, comme on l'a toujours été, comme tout le reste de l'Italie centrale, profondément communiste et militant...

\section{II - LE MÉTIER ET LA RÉUSSITE}

Nouveau paradoxe en effet : même si les conditions politiques nationales étaient défavorables aux forces de gauche $\left({ }^{3}\right)$, on comprend mal comment, au moment de l'opération de décentralisation des années cinquante, les ouvriers du textile, très syndiqués et très combatifs, ont pu finalement accepter leur transformation en producteurs indépendants à la situation précaire. C'est que l'opération, menée sur le mot d'ordre " più di proletari, tutti proprietari ", a joué sur la prégnance sociale, dans la société pratésienne, des valeurs du métier et de l'aspiration au travail indépendant. Le travailleur pratésien, ou toscan, est l'héritier des mezzadri : que ceux-ci soient demeurés fermiers ou qu'ils aient glissé peu à peu vers l'artisanat, ils ont toujours eu à organiser eux-mêmes leur activité. Leurs descendants, ouvriers et artisans du textile dans une cité faiblement et tardivement industrialisée, ont conservé les valeurs du métier. Aussi loin qu'on remonte dans le temps l'idéal social de Prato est toujours le mettersi in proprio (l'installation à son compte).

( $\left.{ }^{1}\right)$ P. Ganugi, M. Romagnoli, Aspetti della domanda e dell'offerta di lavoro nell area pratese, Prato, CCS, 1982.

$\left({ }^{2}\right)$ Précisément on s'enrichit (les chiffres de consommation d'énergie, de construction de maisons individuelles, d'achat d'équipement ménager, etc. sont très supérieurs aux moyennes nationales) parce qu'il est toujours possible à Prato de produire plus, de travailler plus (heures supplémentaires et travail de nuit, travail à domicile en sus du travail salarié, double emploi, travail à domicle des femmes sans profession, etc., souvent sans déclaration). De plus la répartition des revenus est mal connue : en raison de ce qui échappe à la fiscalité d'une part, en raison du cumul des revenus familiaux ensuite. Cf. CENSIS, $\|$ caso Prato : qualità e stili di vita in una società evolutiva, Milano, Etas Libri, 1980; L. Fanti, Redditi, risparmi e consumi delle famiglie dei lavoratori in un' area industrializzata : il caso di Prato, Prato, Camera del lavoro, CGIL, 1983.

$\left({ }^{3}\right)$ L'après-guerre est marquée en Italie par l'exclusion du PCI du gouvernement central et par la reprise en main de tous les pouvoirs par la DC. 
La première ressource sociale à favoriser l'organisation de la diffusion industrielle est donc une culture locale de type productif $\left({ }^{1}\right)$. Étant demeurée monoproductive la ville s'identifie au travail de la laine. Le contact direct avec la matière travaillée est manifeste chez tous: tout Pratésien qui se respecte est capable d'évaluer un tissu du regard et d'en déterminer la composition du bout des doigts. Tant il est vrai que ce savoir-faire est transmis comme un héritage : l'activité lainière, souvent exercée en famille à l'intérieur de la maison familiale, a bercé chacun dès l'enfance. La dextérité dans le travail de la laine, l'intelligence de son commerce y sont des éléments de statut incontestablement classant. Le très haut degré de qualification qui y règne est à la fois porteur de qualité dans la production et d'innovation dans le processus productif. Cette éthique du métier, marque d'un monde d'artisans, ne se comprend qu'au regard de l'aspiration au travail indépendant. Après la décentralisation forcée de 1950 la multiplication rapide des installations d'artisans et des carrières d'impannatori en atteste : l'opération a été relayée par une dynamique sociale. Car réussir, à l'aune traditionnelle de cette société, c'est être un travailleur indépendant, celui qui produit à son compte et celui qui sait vendre. Monde d'artisans et de marchands où les figures mythiques sont les grands marchands médiévaux et les impannatori aux fortunes fulgurantes : la mémoire collective entretient soigneusement leur légende.

Dans le vécu des acteurs sociaux l'installation à son compte, fréquente parmi les ouvriers et les employés, est un motif de visible fierté. Certains, à la trajectoire plus précaire, recommencent cette installation deux ou trois fois, retournant entre temps à leur situation d'origine et consentant de lourds sacrifices : cette " auto-exploitation " atteste de la force de la représentation sociale de l'indépendance professionnelle. Aussi les travailleurs indépendants (artisans, commerçants) sont-ils exceptionnellement nombreux à Prato : 27\% des actifs, soit le double de l'Italie centrale en général pourtant terre de travailleurs indépendants $\left({ }^{2}\right)$. S'installer à son compte est plus facile qu'ailleurs dans le système Prato : point n'est besoin de trop de capitaux; importent plus une bonne connaissance du textile et un bon réseau de relations locales. Cette aspiration n'est crédible cependant que dans le cadre de l'organisation familiale du travail.

La tradition "mezzadrile" de coopération de tous les membres de la famille dans le travail s'est maintenue à Prato comme dans toute la Toscane.

(1) Culture s'entend ici au sens ethnologique du terme, ensemble de normes et de comportements reconnus et transmissibles. Local s'entend comme désignant l'espace défini par ces règles du jeu. On verra ailleurs (cf. E. Ritaine, 1987) comment les systèmes industriels locaux peuvent être, en Italie, traités comme des espaces sociaux réels sans pour autant tomber dans la monographie.

$\left({ }^{2}\right)$ Le circondario de Prato compte 92355 actifs dont 60230 dans l'industrie (essentiellement le textile-confection) : parmi ceux-ci $4 \%$ sont entrepreneurs-professions libérales, $26,5 \%$ travailleurs indépendants, $11,6 \%$ cadres-employés, $57,1 \%$ travailleurs dépendants (ISTAT, 1981 ). 
En effet, la famille toscane est de type famille étendue où, si la cohabitation de plusieurs générations qui sert de critère à ce type ne s'est pas toujours maintenue, les rapports de parentèle sont extrêmement denses et structurants $\left({ }^{1}\right)$. A Prato, où l'entreprise est familiale, seule la mise en commun des revenus (du travail officiel, du travail irrégulier, de l'économie domestique) peut permettre d'accéder un jour à une installation indépendante : le groupe sert de banque et assure la première main-d'œuvre. La division du travail habituelle au sein de la famille attribue aux hommes chefs de famille les emplois officiels ou le rôle d'entrepreneur, aux femmes, aux jeunes et aux personnes âgées les rôles de collaborateurs occasionnels et de travailleurs non déclarés. La famille est un groupe économique à la réussite duquel chacun se doit de participer : à ce titre elle apparaît comme l'agent économique de base le plus important. Sans les stratégies familiales la multiplication des petites entreprises serait impossible; sans les alliances entre familles les réseaux de coopération entre petits producteurs ne seraient pas si sûrs; sans la protection familiale, pour ceux que l'instabilité du marché du travail malmène, les tensions sociales seraient trop fortes. La famille pratésienne, généralement la famille dans toutes les régions d'économie diffuse en Italie, évoquent l'analyse de J. Schumpeter lorsqu'il écrivait que c'est la famille qui est le véritable sujet d'une analyse de mobilité sociale $\left({ }^{2}\right)$.

La diffusion industrielle s'est très fortement structurée à Prato grâce aux dispositions de cette société locale. Ce faisant elle a transformé cette société, bien au-delà des changements morphologiques. Tout en réactualisant valeurs et pratiques d'un monde d'artisans, elle les a tirées vers celles d'un monde d'entrepreneurs : réussir ce n'est plus seulement s'installer à son compte, c'est aussi réussir en termes capitalistes; réussir ce n'est plus seulement occuper une position valorisée dans la société locale, c'est aussi se situer dans les rapports de force de la société globale. En transformant profondément l'organisation économique, la diffusion industrielle a ouvert la voie à une forte aspiration à l'ascension sociale : celle-ci est devenue le processus social dynamisant, celui qui active les dispositions sociales des acteurs. Ceux-ci ont pris rapidement conscience de la fluidité sociale qu'instaurait la structuration du système : jusque dans les années soixante-dix en effet, il semble y avoir eu des possibilités d'ascension sociale. La catégorie des entrepreneurs (chefs d'entreprise et impannatori) s'est constituée par ouverture sociale : dans toutes les zones d'économie diffuse l'origine sociale des entrepreneurs est plus diversifiée qu'ailleurs; les entrepreneurs de Prato sont majoritairement en ascension sociale individuelle $(66 \%$ d'entre eux ont exercé une activité antérieure;

( $\left.{ }^{1}\right)$ En Italie en $1971,16,9 \%$ des familles sont des familles étendues. En Toscane elles sont $25,3 \%$. Les régions de diffusion industrielle correspondent aux régions de familles étendues. Cf. M. Paci (a cura di), Famiglia e mercato del lavoro in un' economia periferica, Milano, Angeli, 1983.

$\left({ }^{2}\right)$ J. Schumpeter, Impérialisme et classes sociales, Paris, Ed. Minuit, 1972. 
parmi ceux-ci $44 \%$ ont été employés, $16,7 \%$ artisans, $10,6 \%$ ouvriers) ${ }^{1}$ ). La multiplication des installations artisanales de la part d'ouvriers et d'employés constitue elle aussi un processus d'ascension sociale, même si la fréquence des faillites en atteste la précarité $\left({ }^{2}\right)$. Les réussites qui ont marqué la période de structuration du système ont ainsi accru la "plausibilité " $\left({ }^{3}\right)$ de l'aspiration à l'ascension sociale dans la société pratésienne.

Peut-être y a-t-il là un effet pervers car, passé la période de construction du système, les positions semblent s'être à nouveau figées et les possibilités d'ascension sociale restreintes. Une analyse des trajectoires familiales, sous l'angle des générations, le montre bien. Dans la catégorie des entrepreneurs par exemple $\left({ }^{4}\right)$ le pourcentage de fils d'ouvriers devenus entrepreneurs est fort parmi les entrepreneurs de plus de 60 ans (50\%), mais il diminue très vite ensuite : cette ouverture correspond sans doute à la période de décentralisation des années cinquante. Ensuite ce sont des fils d'artisans et des fils d'employés qui sont devenus entrepreneurs; aujourd'hui la quasi-totalité des entrepreneurs de $20-30$ ans est formée d'héritiers $(83,3 \%)$. La catégorie des artisans s'est elle aussi transformée : elle n'est plus qu'une catégorie d'arrivée. Ouvriers et employés continuent à y entrer, mais le groupe s'est stabilisé et professionnalisé au point de représenter une réussite en soi : les fils d'artisans demeurent artisans $\left({ }^{5}\right)$. La maturité industrielle du système limite donc désormais l'ouverture sociale alors que la motivation principale des acteurs demeure l'ascension sociale : la prise de conscience de ce phénomène pourrait priver le système de son principal atout. Déjà les efforts à déployer pour réussir sont si grands que les valeurs du métier sont atteintes de ce qu'on nomme à Prato "monetarizzazione " : la course effrénée à la production est une course

( $\left.{ }^{1}\right)$ P. Bartolini-Nerini, A. Miliotti Gentile, Prato, singolarità di un modello di piccola-media impresa, Firenze, Le Monnier, 1983.

${ }^{(2)}$ On ne dispose pas pour l'heure d'un travail sociologique sur les artisans pratésiens et leurs trajectoires. Les associations d'artisans devraient cependant être bientôt en mesure de fournir ces données.

$\left({ }^{3}\right)$ D'après P. C. Berger, T. Luckmann, The social construction of reality, Harmondsworth, Penguin Books, 1981

$\left({ }^{4}\right.$ ) Activité professionnelle du père selon l'âge de l'entrepreneur interviewé (source : BartoliniNerini, op. cit.).

\begin{tabular}{lccccc}
\hline \multicolumn{1}{c}{$\%$} & $20 / 30$ & $30 / 40$ & $40 / 50$ & $50 / 60$ & +60 \\
\hline Entrepreneurs & 83,3 & 52,6 & 42,3 & 28,6 & 25,0 \\
Travailleurs indépendants & 0 & 31,6 & 30,8 & 21,4 & 18,7 \\
Employés & 16,7 & 7,9 & 11,5 & 21,4 & 12,5 \\
Ouvriers & 0 & 7,9 & 15,4 & 28,6 & 49,7 \\
\hline \multicolumn{1}{c}{ Total } & 100 & 100 & 100 & 100 & 100 \\
\hline
\end{tabular}

(5) On remarque également qu'un fort pourcentage de fils d'employés deviennent entrepreneurs. Il semble s'agir d'une catégorie de passage, accumulant sans doute un capital utile lié à sa position professionnelle (savoir-faire et relations sur le marché local). 
à l'accumulation financière où se perdent peu à peu l'éthique du travail et l'épanouissement de l'artisan dans son œuvre; trop souvent désormais un acteur est classé par ses consommations ostentatoires plus que par ses capacités professionnelles.

Dérive de l'aspiration contemporaine, dérive des valeurs héritées d'autant plus inquiétantes que la stratification sociale pratésienne s'est faite sur des lignes de classification par âge, par sexe et par origine géographique qui relèguent les plus démunis. La centralité socio-économique est occupée par des hommes d'âge mûr, d'origine exclusivement pratésienne et toscane : chefs d'entreprise, impannatori, commerçants et artisans importants, il s'agit de l'aristocratie productive locale. Ensuite les petits artisans et les ouvriers très qualifiés constituent un cercle intermédiaire numériquement important. Enfin l'extrême périphérie est formée des ouvriers non qualifiés et des travailleurs à domicile. Là les facteurs de marginalisation se cumulent : on y est plus souvent d'origine méridionale, on y est plus souvent de sexe féminin, on y travaille plus souvent au noir. Les immigrés méridionaux de la première génération, voire les paysans toscans attirés par l'expansion pratésienne, se sont remarquablement bien intégrés : émigrant pour raisons économiques ils ont trouvé dans cette ville obsédée par la réussite productive une occasion d'améliorer leur sort. Mais la deuxième génération, plus scolarisée, plus consciente des limites assignées à son avenir dans ce système, commence à refuser son sort. Le système tend donc à avoir des problèmes de renouvellement de sa main-d'œuvre de base et on s'y interroge sur la façon de revaloriser le travail ouvrier.

Le système n'en est sans doute pas pour autant menacé dans l'immédiat : tout au plus sa reproduction est-elle un peu plus problématique. En effet si l'aspiration commune à l'ascension sociale est de plus en plus difficilement satisfaite dans le système, le degré de sécurité économique qu'il continue à assurer à la plupart dans une période de crise et de chômage (et même si ce résultat est obtenu en partie grâce au travail irrégulier) assure toujours un bon consensus en sa faveur $\left({ }^{1}\right)$. De plus la culture locale du travail et de la production est si profondément enracinée, si bien relayée par la socialisation familiale, qu'elle demeure un terrain très stable pour l'organisation de la diffusion industrielle. Les valeurs centrales, valeurs héritées ou aspirations, s'émoussent pourtant dans le changement social : le système a, symtômatiquement, de plus en plus besoin de la régulation communautaire qu'assure la trame des réseaux locaux.

(1) Dans les conjonctures récentes les plus mauvaises le chômage à Prato a atteint plus ou moins $7 \%$ des actifs (les jeunes et les femmes en sont les principales victimes). En Italie le taux est de $11 \%$; de $20,3 \%$ en Sardaigne, $15,1 \%$ en Sicile, etc. (1984, INSEE). Cf. D. Berardi, M. Romagnoli, L'area pratese tra crisi e mutamento, Prato, CCS, 1982. 


\section{III - LA TRAME RÉTICULAIRE}

L'ultime paradoxe de Prato n'en est peut-être pas un, à y bien regarder. Cette cité hyperproductiviste est une ville traditionnellement communiste, partie intégrante de la subculture politique "rouge " qui caractérise l'Italie centrale : elle vérifie parfaitement l'hypothèse selon laquelle plus une subculture politique est forte, plus la régulation locale est efficace $\left({ }^{1}\right)$. La subculture politique "rouge " a pour trait principal l'intensité des réseaux de toutes natures (interrelations, alliances familiales, voisinage, associations, regroupements idéologiques) qui assurent une régulation civile puissante : les sociétés de subculture "rouge " sont d'abord des sociétés réticulaires, la coloration idéologique ne prend sens qu'ensuite, par rapport aux réseaux. L'organisation de la nébuleuse pratésienne ne se comprend qu'ainsi : là où l'extrême dispersion productive aurait pu aboutir au chaos, des réseaux préexistants assurent la circulation de l'information et des biens, la coopération des uns et des autres, les règles de la concurrence entre les différents acteurs. Ainsi les affaires se font-elles entre membres de familles alliées ou entre connaissances de longue date; ainsi le contrôle social est-il suffisamment fort pour qu'il soit difficile de déroger aux normes en vigueur; ainsi les rapports de force se rééquilibrent-ils en grande partie. La société locale ploie le fonctionnement du marché à ses propres règles : la diffusion industrielle trouve une nouvelle ressource dans l'organisation collective pratésienne, ressource de stabilisation et de reproduction cette fois.

Tradition réticulaire et nécessité fonctionnelle se conjuguent pour constituer à Prato un tissu social à la trame particulièrement dense. Le taux de participation aux organisations professionnelles (syndicats et associations) par exemple est un des plus élevés en Italie (sur 1000 actifs 864 inscrits, contre 619 en Emilie-Romagne qui est la région la plus militante, et une moyenne nationale de 392) $\left({ }^{2}\right.$ ). La présence sociale des partis de gauche (du PCI surtout) et des syndicats (la CGIL communiste surtout) canalise très efficacement la sociabilité locale : ils sont présents sur tous les terrains, accompagnés par une multitude d'associations de même orientation (dont la très puissante ARCI,

( $\left.{ }^{1}\right)$ La Toscane est un des principaux bastions du vote communiste; la particularité des subcultures, qu'elles soient " rouges " ou "blanches " (comme en Vénétie par exemple) est de manifester une orientation idéologique stable et partant un choix électoral stable et homogène, depuis la guerre. Pour donner une idée de l'ampleur du phénomène on peut noter qu'aux élections régionales de 1985 le score national du PCI est de 30,8\% suffrages exprimés; celui de la DC est de $34,7 \%$. En Vénétie, le PCI rassemble $22,1 \%$ suffrages, la DC $42,7 \%$; en Toscane $\mathrm{PCI}=46,7 \%, \mathrm{DC}=26.4 \%$. A Prato, $\mathrm{PCI}=48,4 \%$ (encore est-il en recul sur les précédents résultats pratésiens, au profit du PSI et des listes écologiques); $\mathrm{DC}=28 \%$; PSI $=11,6 \%$. Sur les subcultures politiques italiennes cf. C. Trigilia, Sviluppo economico e trasformazioni sociopolitiche dei sistemi territoriali e economia diffusa. Le subculture politiche territoriali, Milano, Quaderni della Fondazione Feltrinelli, 1981; C. Trigilia, "La regolazione localista : economia e politica nelle area di piccola impresa », Stato e Mercato, 1985, 14.

( ${ }^{2}$ ) CENSIS, 1980, op. cit. 
Associazione ricreativa culturale italiana), maîtres des institutions locales (la plupart des municipalités de l'agglomération, la province, la région Toscane). Comme souvent en Italie l'ancien municipalisme a été relayé par un vote si stable qu'il est devenu un vote d'identité : Prato est une communauté idéologique, elle a su devenir, en outre, une communauté productive $\left(^{1}\right)$.

Car les rapports de force sur le marché local sont considérés comme la seule affaire des Pratésiens (un investisseur étranger par exemple est inimaginable), et régulés par eux selon leurs propres règles : la régulation sociopolitique y est strictement locale. Aussi les relations industrielles, dans un premier temps perturbées par la décentralisation industrielle, se sont-elles recomposées autrement. Le syndicat a appris à fragmenter son action pour l'adapter à un mode de production lui-même fragmenté $\left({ }^{2}\right)$. Présent de droit dans les grandes entreprises grâce aux consigli di fabbrica, il l'est aussi dans les entreprises artisanales grâce à l'activisme de ses militants, voire auprès des travailleurs à domicile dont il essaie d'assurer la protection. Non content de faire appliquer les conventions régionales ou de branches, il négocie des accords entreprise par entreprise. Et dans la sensibilité communiste ambiante tout salarié le considère comme un interlocuteur naturel et n'hésite pas à consulter à la Camera del lavoro (Bourse du travail). Enfin l'une des deux grandes associations d'artisans, bien que très indépendante, est elle aussi d'inspiration communiste.

Le réseau syndical est ainsi un acteur incontournable de la négociation. Acteur incontournable et incontourné car le patronat local ne peut que se féliciter d'un partenaire suffisamment puissant et pragmatique pour stabiliser les relations industrielles: Prato a l'un des plus faibles nombres d'heures de travail perdues pour fait de grève en Italie. En effet la négociation qui porte sur les salaires pour les ouvriers, sur les tarifs pratiqués par les artisans terzisti (entièrement dépendants des commanditaires) évite le développement de l'exploitation du travail, et donc de tensions sociales. Seule la partie immergée du travail, la plus exploitée (un travailleur à domicile non déclaré peut être sous-payé jusqu'à $1 / 5$ du salaire en usine) échappe à ce contrôle. L'originalité des relations industrielles à Prato tient à une différence de nature

( ${ }^{1}$ La culture communiste qui est une culture du travail et de la production, constitue dans ce cas une expression en cohérence avec l'éthique locale de la production.

$\left({ }^{2}\right)$ Contrairement à ce qu'affirme trop souvent la presse française, les régions de diffusion industrielle sont des régions où le syndicalisme est fort et où il contribue à stabiliser les rapports de force. Alors que le syndicalisme est en crise au nord de l'Italie, dans les zones de grande industrie, il progresse dans les régions de diffusion industrielle. Il ne reste faible que dans les entreprises artisanales, mais il faut tenir compte de la présence sociale des syndicats qui va bien au-delà des chiffres. Les enquêtes menées en 1982 en Vénétie (à Bassano) donnent $48 \%$ des actifs syndiqués dans les PME, $17 \%$ dans les entreprises artisanales; en Toscane (Valdelsa) $80 \%$ des actifs dans les PME, $41 \%$ dans les entreprises artisanales. 45\% des entreprises de Bassano, $60 \%$ de celles du Valdelsa sont concernées par des négociations directes avec le syndicat (A. Bagnasco, C. Trigilia, 1984, 1985, op. cit.). A Prato, environ $60 \%$ des salariés de l'industrie textile sont syndiqués. 
fortement marquée entre les acteurs de la négociation : d'un côté des réseaux de gauche, constituant presque toute la trame de la société locale, exerçant un énorme pouvoir civil; de l'autre un groupe d'entrepreneurs (l'UIP, l'union industrielle) et de financiers, d'orientation démocrate-chrétienne : alors que ce groupe assure le pilotage industriel du système il n'a pas de présence sociale, mais un énorme pouvoir financier $\left({ }^{1}\right)$.

L'autre originalité de ces relations industrielles localistes est d'associer à la négociation les acteurs politiques locaux, dans le rôle d'auxiliaires : fédérations de partis, municipalités, élus régionaux ont pour fonction politique essentielle de faciliter ces relations industrielles. Leurs bons offices sont sollicités quand la négociation se bloque; ils organisent des colloques rassemblant tous les partenaires économiques; ils financent des centres de recherche socio-économiques; ils assurent la promotion à l'extérieur d'une ville qui se veut moderne, productive, innovatrice. Leur fonction n'est donc que complémentaire : toute mise sur pied d'une politique publique est impossible à Prato si elle menace si peu que ce soit d'entraver la production $\left({ }^{2}\right)$. Ainsi aperçoit-on les véritables effets pervers de la diffusion industrielle pratésienne : les effets pervers atteignant la collectivité ne sont pas négociables si leur effacement semble risquer de gêner la production (et l'on entend bien qu'il peut s'agir d'un risque fantasmatique). Les conditions de travail par exemple sont plus difficiles à négocier que les salaires: l'ajustement des conditions de sécurité et de salubrité, la réduction de la durée du travail sont des transformations que beaucoup de petits producteurs ne pourraient pas supporter sur le plan financier. Il semble y avoir en conséquence une sorte de consensus silencieux sur ce dossier : les syndicats pratésiens n'ont jamais réussi à mobiliser sur les thèmes de la santé au travail, pourtant particulièrement dramatiques ici (vieillissement précoce de certains salariés dû à la tension nerveuse de la course à la productivité, accidents du travail nombreux par non-respect des normes de sécurité, maladies professionnelles graves et endémique, surtout sur les postes où sont manipulés des produits chimiques dangereux, comme la carbonisation des chiffons et la teinturerie).

Les effets de la diffusion industrielle sur l'environnement sont un autre dossier épineux. Née d'une multitude de décisions individuelles non concertées, la diffusion industrielle a mité le milieu urbain d'installations productives,

( ${ }^{1}$ ) La disproportion entre pouvoir civil et pouvoir financier est telle qu'on entend certains militants communistes suggérer la création d'une banque contrôlée par les communistes. A noter que la région Toscane (socialo-communiste) a créé et contrôle un organisme régional de crédit aux artisans (Fiditoscana).

( ${ }^{2}$ ) Il faut préciser que le fait tient d'abord à la faiblesse des ressources financières des municipalités italiennes. Néanmoins la volonté politique joue aussi un rôle : certaines municipalités, plus interventionnistes comme celle de Bologne, certaines régions, comme l'Emilie-Romagne ou les Marches, parviennent à développer des politiques publiques d'accompagnement du développement économique. 
induisant une urbanisation anarchique : installations productives et habitat imbriqués, voies de circulation défoncées par les poids lourds, spéculation immobilière sauvage, pollution des eaux (par les teintures) et de l'air (par la poussière de laine), etc. Là encore toute politique publique réformiste serait difficilement admise puisqu'elle entraverait la libre décision des acteurs économiques. Étrange communauté locale en définitive, dotée d'une forte identité idéologique, capable de se comporter en communauté productive en régulant ses tensions intérieures, mais comme empêchée de prendre en compte l'intérêt général.

Ou bien est-ce encore un effet des valeurs de base de la société pratésienne? Si celle-ci se comporte comme une communauté, c'est uniquement lorsqu'il s'agit de protéger les règles d'un jeu considéré comme satisfaisant les stratégies individuelles-familiales : l'individualisme familial, si l'on peut risquer ce terme, l'emporte toujours, empêchant par exemple le développement d'un mouvement coopératif, plus soucieux de l'intérêt général, comme en Émilie-Romagne. Aussi toutes les demandes réformistes qui montent face aux difficultés de reproduction du système se heurtent-elles à une question fondamentale : peut-on, dans ces systèmes industriels où le facteur de production le plus important est la société locale même, rationaliser le désordre sans anéantir le potentiel de créativité? Ou bien la société locale dégagera-t-elle d'elle-même de nouvelles ressources? Tout l'intérêt d'observer à présent la maturité des systèmes de diffusion industrielle est là.

\section{EVELYNE RITAINE}

Fondation nationale des sciences politiques. Institut d'études politiques de Bordeaux. 